\title{
Capacidade de Regeneração de Egeria densa nos Reservatórios de Paulo Afonso, BA ${ }^{1}$
}

\author{
Regeneration Capacity of Egeria densa in Reservoirs in Paulo Afonso, Bahia
}

OLIVEIRA, N.M.B. ${ }^{2}$, SAMPAIO, E.V.S.B. ${ }^{3}$, PEREIRA, S.M.B. ${ }^{4}$ e MOURA JUNIOR, A.M. ${ }^{5}$

\begin{abstract}
RESUMO - Para controlar a proliferação de Egeria densa e reduzir os prejuízos que vêm causando na geração de energia em Paulo Afonso, é necessário conhecer sua capacidade de regeneração. Para isso, foram marcados quadrados de $4 \mathrm{~m}^{2}$, em diferentes épocas do ano e em três profundidades (0-2, 2-6 e 6-9 m), e retiradas as biomassas de $E$. densa iniciais e três meses depois. Na profundidade de $0-2 \mathrm{~m}$, as retiradas de plantas foram repetidas por cinco vezes, em quatro dos mesmos quadrados. Nesta profundidade, as massas regeneradas $\left(518 \mathrm{~g} \mathrm{~m}^{-2}\right)$ foram semelhantes às iniciais $\left(482 \mathrm{~g} \mathrm{~m}^{-2}\right)$, independentemente da época do ano (estação seca, geral: $506 \mathrm{~g} \mathrm{~m}^{-2}$; chuvosa: $513 \mathrm{~g} \mathrm{~m}^{-2}$ ) e da repetição da retirada. Na de 2-6 m,

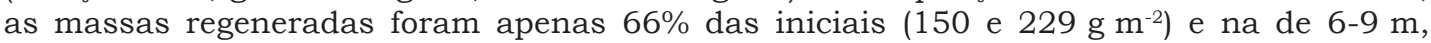
apenas $21 \%$ (11,6 e 54,9 $\left.\mathrm{g} \mathrm{m}^{-2}\right)$. A rápida recolonização e o grande crescimento de $E$. densa tornam muito dificil erradicá-la dos reservatórios do São Francisco. Sugere-se que seja explorado esse grande potencial de produção de biomassa.
\end{abstract}

Palavras-chave: biomassa, colheita das plantas, profundidade da água, crescimento sazonal.

\begin{abstract}
The regeneration capacity of Egeria densa must be studied to control its proliferation and reduce the damage to energy generation at Paulo Afonso power plant. Thus, $4 \mathrm{~m}^{2}$ plots were established in different seasons and at three water depths (0-2, 2-6, and 6-9 $\mathrm{m}$ ) with removal of the initial plant biomass and three months later. At 0-2 $m$ depth, removal was repeated five consecutive times in four of the plots. At this depth, regenerated biomass $\left(518 \mathrm{~g} \mathrm{~m}^{2}\right)$ was similar to the initial one $\left(482 \mathrm{~g} \mathrm{~m}^{2}\right.$ ), regardless of season (dry season, overall: $506 \mathrm{~g} \mathrm{~m}^{2}$; rainy season: $513 \mathrm{~g} \mathrm{~m}^{2}$ ) and repeated removal. At 2-6 $\mathrm{m}$, regenerated biomass was only 66\% of the initial ones $\left(150\right.$ and $\left.229 \mathrm{~g} \mathrm{~m}^{2}\right)$ and at 6-9 $\mathrm{m}$, only $21 \%\left(11.6\right.$ and $\left.54.9 \mathrm{~g} \mathrm{~m}^{2}\right)$. The rapid re-colonization and growth of $\boldsymbol{E}$. densa makes it very difficult to eradicate it from São Francisco River reservoirs. It is suggested that this great biomass production potential be put to use.
\end{abstract}

Key words: biomass, plant harvesting, water depth, seasonal growth.

\section{INTRODUÇÃO}

Egeria densa, pertencente à família Hydrocharitaceae, conhecida popularmente como "elodea brasileira", é uma macrófita aquática de ambiente limnético, submersa, enraizada, nativa da América do Sul e amplamente disseminada por várias partes do mundo, principalmente em regiões de clima tropical. Em muitos países é considerada uma invasora agressiva, formando uma cobertura vegetal densa, muitas vezes monoespecífica (Winton $\&$ Clayton, 1996). No Brasil, é considerada como nativa da região Sudeste, em Minas Gerais e no Espírito Santo (Cook \& Urmi-König, 1984), mas encontra-se em corpos d'água de todas as

1 Recebido para publicação em 3.3.2005 e na forma revisada em 8.5.2005 .

Parte do projeto de pesquisa: Estudo do ecossistema dos reservatórios do sistema hidroelétrico de Paulo Afonso e Itaparica (Terceira Etapa).

2 Bióloga, Aluna do PPGB, UFRPE, <nmboliveira@bol.com.br $>;{ }^{3}$ Prof. do Dep. de Energia Nuclear, UFPE. Av. Prof. Luís Freire 1000, 50740-540 Recife-PE, <esampaio@ufpe.br>; ${ }^{4}$ Prof. do Dep. de Biologia, UFRPE, <soniabp@terra.com.br>; ${ }^{5}$ Prof. Colégio de Aplicação, UFPE, <alfmoura@cap.ufpe.br>. 
regiões. Como outras Hydrocharitaceae, tem uma capacidade fotossintética alta, módulos vegetativos de tamanho pequeno, associados a baixo custo energético de construção, boa capacidade de dispersão de fragmentos vegetativos e alta sobrevivência (Kahara \& Vermaat, 2003).

Apesar de, no Brasil, ocorrerem extensas áreas cobertas por macrófitas aquáticas na maioria dos ecossistemas aquáticos limnéticos, ainda são escassos os estudos sobre a produtividade dessas comunidades. Em parte, isso pode ser atribuído à idéia comum, no passado, de que o papel desses vegetais nos ecossistemas era insignificante. Têm pesado, também, as dificuldades metodológicas na determinação da biomassa e da produtividade primária dos diferentes grupos ecológicos. Nas duas últimas décadas, tem aumentado o interesse por esses estudos, devido à construção de represas, principalmente, para geração de energia elétrica (Esteves, 1982; Menezes, 1984; Menezes et al., 1993; Camargo \& Esteves, 1996; Nascimento, 2002).

Nos reservatórios do Sistema Hidroelétrico de Paulo Afonso e Itaparica, no rio São Francisco, há algum tempo, E. densa vem se proliferando, causando prejuízos na geração de energia, devido ao seu acúmulo nas grades de contenção das turbinas (Borges Neto \& Pitelli, 2004; Mendes et al., 2004). Este problema também ocorre em hidrelétricas de outras regiões (Barreto et al., 2000; Marcondes et al., 2003; Borges Neto \& Pitelli, 2004). As condições de crescimento, em Paulo Afonso e Itaparica, parecem muito favoráveis à proliferação de E. densa, tanto em termos de substrato, nutrientes e luminosidade, quanto pela ausência de predadores e de espécies competidoras. Recentemente, foi observado que todas as áreas nos reservatórios propícias ao seu desenvolvimento, bem como as áreas ricas em substrato lamoso e com profundidade até $9 \mathrm{~m}$ (Nascimento, 2002), estavam cobertas com E. densa.

Em razão dos prejuízos na geração de energia, a CHESF, em parceria com a UFRPE, iniciou estudos com o objetivo final de controlar o crescimento de $E$. densa nos reservatórios e/ou aproveitar a massa produzida para algum fim lucrativo. Parte desses estudos foi a determinação da capacidade de regeneração da vegetação depois que as plantas de uma área eram arrancadas, o que constitui o objetivo específico deste trabalho.

\section{MATERIAL E MÉTODOS}

A área de estudo é um trecho da bacia hidrográfica do rio São Francisco, onde está o Complexo Hidroelétrico de Paulo Afonso, no município de mesmo nome $\left(09^{\circ} 21^{\prime} 42^{\prime \prime} \mathrm{S}\right.$ e $38^{\circ} 16^{\prime} 19^{\prime \prime} \mathrm{W}$ Gr.), na Bahia, sendo constituído pelos reservatórios de Delmiro Gouveia e Apolônio Sales (Silva, 1985; Nascimento, 2002). No Delmiro Gouveia, foram escolhidas duas estações de trabalho (casa da bomba e dreno de areia da Usina II) e, no Apolônio Sales, uma estação (barragem móvel). Nas estações, foram marcadas áreas para trabalho, nas profundidades de 0-2, 2-6 e 6-9 m, e as marcações e coletas foram realizadas entre novembro de 2001 e outubro de 2003.

Em cada área, foram marcados quadrados de $4 m^{2}(2 \times 2 \mathrm{~m})$, delimitados por estacas fincadas no substrato. Posteriormente, procedeuse à retirada manual de toda a biomassa de $E$. densa presente no quadrado, acima do sedimento, trazendo consigo, eventualmente, parte do seu sistema radicular. A primeira biomassa coletada foi considerada como a biomassa inicial (biomassa-estoque). Nos primeiros quadrados, marcados na profundidade de 0-2 m, o estabelecimento de E. densa foi acompanhado visualmente, a cada 15 dias, até que toda a área estivesse ocupada e as plantas estivessem com altura semelhante à das plantas localizadas no exterior do quadrado. Como esse tempo foi de três meses, estabeleceu-se esse período como padrão para a recuperação de todas as demais áreas e profundidades. Findos os três meses, toda a biomassa foi retirada de forma semelhante à descrita anteriormente, sendo considerada a biomassa produzida. Por ocasião de cada uma dessas coletas, foi também retirada a biomassa de E. densa de um quadrado medido ao lado do quadrado marcado, para permitir a comparação não só com a biomassa inicial, mas também com a biomassa presente onde a vegetação não havia sido arrancada anteriormente. Em quatro quadrados, na profundidade de $0-2 \mathrm{~m}$, o processo de retirada da biomassa foi repetido, por quatro períodos consecutivos de três meses. O número de quadrados nas áreas variou em função da 
facilidade de acesso, sendo no mínimo quatro nas profundidades maiores e no máximo sete na menor profundidade. As biomassas coletadas foram secas ao sol no próprio local e, então, conduzidas à UFRPE, onde foram secas em estufa a aproximadamente $70{ }^{\circ} \mathrm{C}$, até alcançar peso seco constante.

A fim de comparar as massas iniciais e finais, em cada profundidade, e também para comparar as massas de cada uma das quatro épocas de regeneração com a massa inicial, foi usado o teste de $t$, pareando-se os dados do mesmo local. Na comparação das massas da estão seca (agosto a janeiro) com as da estação de chuvas (fevereiro a julho) foi usado o teste de $t$ sem pareamento, já que provieram de locais distintos. Para comparar as massas iniciais das três profundidades, foi feita uma análise de variância, considerando um delineamento completamente casualizado. As médias foram comparadas pelo teste de Tukey a $5 \%$ de probabilidade.

\section{RESULTADOS E DISCUSSÃO}

Na profundidade de 0 a $2 \mathrm{~m}$, as plantas de Egeria densa tiveram uma grande capacidade de se restabelecerem e de crescerem, até alcançar massa semelhante à original ou à de áreas onde as plantas não foram perturbadas. O acompanhamento visual de parcelas, montadas em época de água limpa, mostrou que as plantas recolonizaram o espaço vazio em poucas semanas. Uma quinzena após a área dos quadrados ter sido esvaziada de plantas, ela já estava com $30-40 \%$ cobertos por novos ramos de $E$. densa e, em três meses, já estava com cerca de $100 \%$ de reocupação. Ou seja, visualmente, não parecia haver diferença de tamanho e densidade entre as plantas de dentro e de fora dos quadrados. Testando o crescimento de E. densa em tanques, na Nova Zelândia, a partir de pedaços de caules, a ocupação total também foi obtida após três meses (Hofstra et al., 1999).

A recolonização não se deu pela chegada de sementes que, aparentemente, não são produzidas fora da área de origem da espécie (Winton \& Clayton, 1996), e sim pela chegada de fragmentos dos caules da macrófita, trazidos pelas águas, que produzem raízes adventícias e fixam-se no substrato. A velocidade de ocupação da área parece ter sido maior em locais onde o sedimento se apresentava constituído por uma camada profunda de substrato lamoso e menor onde a camada era mais rasa e/ou mais arenosa. É possivel que algumas plantas tenham crescido a partir da brotação de gemas de estolões que permaneceram enterrados no substrato quando elas foram arrancadas. Esse processo não foi observado, mas pode ter sido mascarado pelo crescimento mais rápido das plantas originadas dos fragmentos de caule.

A observação visual foi comprovada pelos resultados das massas de plantas nas parcelas (Figura 1). As massas das novas plantas estabelecidas nos quadrados da profundidade

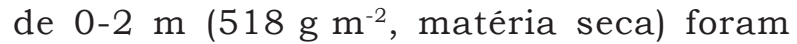
semelhantes às massas iniciais nos mesmos quadrados $\left(482 \mathrm{~g} \mathrm{~m}^{-2}\right)$ e também semelhantes às massas das plantas não-perturbadas, ao lado dos quadrados que haviam sido esvaziados $\left(497 \mathrm{~g} \mathrm{~m}^{-2}\right)$. Isso mostra que três meses foram suficientes para o estabelecimento de novas plantas e para que estas se desenvolvessem até atingir porte semelhante ao das não-perturbadas.

Nas duas profundidades maiores, 2-6 e 6-9 $\mathrm{m}$, as capacidades de reinstalação e crescimento das plantas foram menores que as das plantas que cresceram mais próximas da superficie (Figura 1). Na profundidade intermediária $(2-6 \mathrm{~m})$, as novas plantas atingiram uma densidade semelhante à inicial, porém só produziram $66 \%$ da massa que tinham as

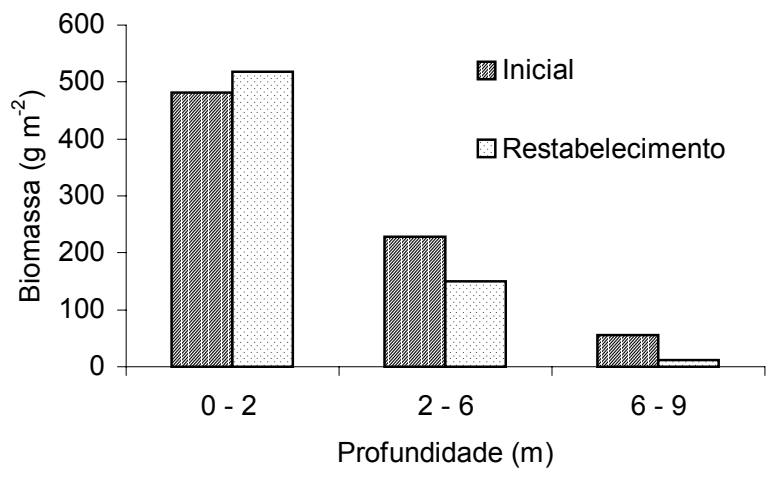

Figura 1 - Biomassa inicial e biomassa restabelecida de Egeria densa três meses após a completa retirada da biomassa inicial, em parcelas situadas em diferentes profundidades, nos reservatórios do Complexo Hidroelétrico de Paulo Afonso, BA.

Planta Daninha, Viçosa-MG, v. 23, n. 2, p. 363-369, 2005 
plantas que ocupavam o mesmo quadrado antes de serem arrancadas $\left(229 \mathrm{~g} \mathrm{~m}^{-2}\right.$ de massa inicial e $150 \mathrm{~g} \mathrm{~m}^{-2}$ de massa restabelecida).

Nos locais mais fundos (6-9 m), mesmo antes de qualquer perturbação, nem toda a área dos quadrados estava coberta por plantas de $E$. densa. Três meses depois que os quadrados foram esvaziados, a nova ocupação era ainda menos densa e as plantas tinham porte menor que antes. Assim, a nova massa formada $\left(11,6 \mathrm{~g} \mathrm{~m}^{-2}\right)$ foi apenas $21 \%$ da massa inicial $\left(54,9 \mathrm{~g} \mathrm{~m}^{-2}\right)$. A causa mais provável da menor recolonização e crescimento, em plantas situadas em maior profundidade, seria a menor intensidade de luz que receberiam, reduzindo a capacidade fotossintética e a acumulação de massa (Bini et al., 1999; Tavechio \& Thomaz, 2003). Mesmo na época de águas mais limpas, a visibilidade ia apenas a cerca de $4 \mathrm{~m}$ de profundidade. Além da luz, poderia haver uma diferença na velocidade do fluxo de água nas diferentes profundidades, que poderia ser menor nas áreas mais rasas, todas mais próximas da borda. Maiores velocidades poderiam impedir que alguns dos fragmentos de caules, originadores de novas plantas, permanecessem parados tempo suficiente para criarem raizes e fixarem-se no sedimento. A velocidade da água é um fator importante na fixação de $E$. densa (Howard Willians et al., 1996; Gantes \& Caro, 2001). Embora não se possa excluir a hipótese de efeito da correnteza, ela fica enfraquecida pela diversidade de locais escolhidos, possivelmente submetidos a correntes distintas.

As produções nas três profundidades, especialmente nas áreas mais rasas, indicam que $E$. densa possui enorme capacidade de acumulação de biomassa, em períodos muito curtos. Poucas espécies vegetais, mesmo as agrícolas, selecionadas pela sua capacidade produtiva, têm capacidade de acumular o equivalente a 5 toneladas de matéria seca por hectare em apenas três meses de crescimento. Produções anuais de E. densa (600-1.000 $\mathrm{g} \mathrm{m}^{-2}$ ) não muito acima das encontradas nos três meses têm sido relatadas em países onde a temperatura varia muito ao longo do ano, como Japão e Estados Unidos (Cook \& Urmi-König, 1984; Haramoto \& Ikusima, 1988; Nakanishi et al., 1989). Entretanto, a produção máxima mensal em pleno verão, quando a temperatura não é limitante, atingiu mais de $400 \mathrm{~g} \mathrm{~m}^{-2}$ no Japão (Haramoto \& Ikusima, 1988) e, nos Estados Unidos, correspondeu a um crescimento que dobrava a biomassa a cada 17 dias (Tanner et al., 1993).

O restabelecimento e o crescimento de E. densa, na profundidade de 0-2 m, foram semelhantes ao longo de todo o ano, sem variações sazonais significativas. Também não variaram significativamente as biomassas dos locais não-perturbados. Como parcelas nãoperturbadas e regeneradas também não diferiram, a média geral das massas de todas elas na estação seca ou de águas limpas foi de $506 \mathrm{~g} \mathrm{~m}^{-2}$, enquanto a da estação de chuvas ou de águas turvas foi de $513 \mathrm{~g} \mathrm{~m}^{-2}$. Essa ausência de variação tem sido relatada para macrófitas enraizadas em represas de outras regiões, como a do Lobo, em São Paulo (Menezes et al., 1993), em contraste com a variação das macrófitas flutuantes. Segundo esses autores, as variações bruscas no nível da água são mais importantes que as mudanças de estações do ano. Nos reservatórios estudados no presente trabalho, os niveis da água oscilaram pouco, regulados pelos reservatórios a montante. Por sua vez, a turbidez da água variou muito e, com ela, a penetração de luz. Se a falta de luz parece ter limitado o estabelecimento e crescimento nas profundidades maiores, também deveria ter limitado na época de águas sujas, mesmo na menor profundidade. No entanto, E. densa tem um sistema fotossintético eficiente e com boa capacidade de absorção de $\mathrm{CO}_{2}$ (Maberly \& Madsen, 2002; Lara et al., 2002; Kahara \& Vermaat, 2003) mesmo em águas turvas, assim como E. najas (Tavechio \& Thomaz, 2003). A ausência de medidas de penetração de luz ao longo do ano - especialmente de luz difusa, que não é indicada pelo disco de Sechi - impede um aprofundamento da questão, neste trabalho.

A medição das biomassas iniciais e de restabelecimento, em quadrados localizados em diferentes áreas, permite uma boa amostragem da variação espacial nos reservatórios; contudo, pode mascarar qualquer queda na capacidade de recolonização de uma área cujas plantas foram arrancadas repetidas vezes. Essa capacidade poderia cair caso a recolonização dependesse da rebrota de estolões da 
área ou se houvesse uma redução na capacidade do substrato de disponibilizar nutrientes para as plantas, com a repetida formação e retirada da biomassa. O acompanhamento por um ano, nos mesmos quadrados, do restabelecimento dos prados arrancados quatro vezes seguidas não mostrou queda significativa da biomassa formada, embora pareça ter havido uma tendência de queda (Figura 2). A tendência é apenas aparente e pode ser explicada pelo fato de dois dos quadrados medidos - os mais próximos a uma das margens - terem ficado cobertos com macrófitas flutuantes alguns meses após sua marcação, permanecendo assim até o fim do período experimental. A eliminação dessas repetições leva as médias das biomassas das plantas que se restabeleceram nos períodos seguidos a ficarem muito mais próximas da inicial, mas preferiu-se manter essas repetições para ressaltar a capacidade de crescimento de E. densa, mesmo em condições de redução luminosa forte, pela cobertura das outras plantas. O fato de E. densa continuar a se estabelecer e a crescer, com produção pouco inferior à de áreas não-cobertas, mesmo abaixo da espessa $(50-70 \mathrm{~cm})$ e fechada camada de vegetação de borda, comprova sua grande capacidade invasora nos reservatórios do São Francisco, assim como tem ocorrido em outros locais ao redor do mundo (Winton \& Clayton, 1996).

A semelhança, ao longo do ano, das biomassas dos locais não-perturbados e o fato de as áreas recolonizadas atingirem massas de

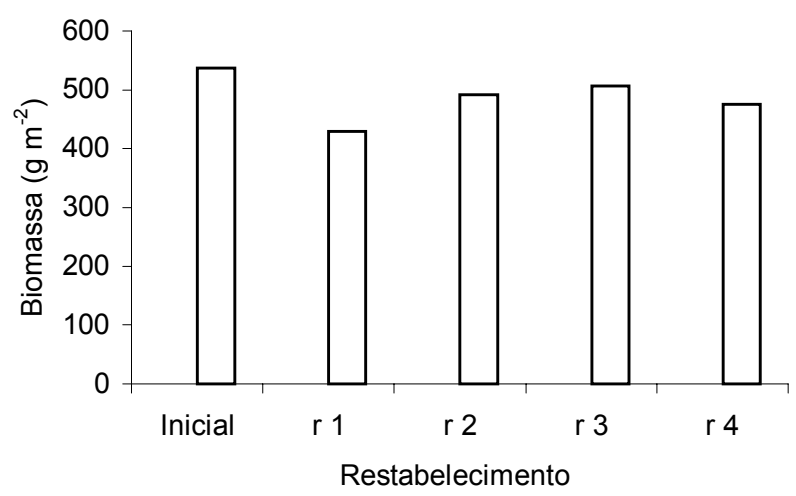

Figura 2 - Biomassa inicial e biomassa restabelecida de Egeria densa em três meses de crescimento, após quatro retiradas consecutivas das biomassas formadas ( $\mathrm{r} 1 \mathrm{ar} 4$ ), em parcelas situadas a 0-2 $\mathrm{m}$ de profundidade, nos reservatórios do Complexo Hidroelétrico de Paulo Afonso, BA. mesma grandeza, pelo menos na profundidade de $0-2 \mathrm{~m}$, indicam que o limite de acumulação parece estar em torno dos $500 \mathrm{~g} \mathrm{~m}^{-2}$. Medidas em outros locais, geralmente em latitudes maiores, têm revelado biomassas apenas um pouco menores (Getsinger \& Dillon, 1984; Feijoó et al., 1996; Wilcock et al., 1999). Atingido o limite de acumulação, a vegetação poderia restringir a produção de biomassa ao necessário para renovação das plantas mortas, das partes desprendidas ou predadas. Resultados do grupo de trabalho da UFRPE (Nascimento, 2002) mostram que o crescimento das plantas continua intenso. As pontas dos ramos de plantas não-perturbadas, na profundidade de $0-2 \mathrm{~m}$, acumularam, a cada 15 dias, uma nova biomassa equivalente a $83 \mathrm{~g} \mathrm{~m}^{-2}$, sem grande diferença ao longo de todo o ano. Acumulando essas produções quinzenais para um período de três meses, as biomassas equivalem a cerca de $500 \mathrm{~g} \mathrm{~m}^{-2}$, valor semelhante ao obtido nas áreas em regeneração do presente trabalho. O trabalho de Wilcock et al. (1999), na Nova Zelândia, também mostra alta capacidade de produção, com taxa de fotossintese bruta de $10-27 \mathrm{~g} \mathrm{~m}^{-2}$ por dia.

Como o estoque de biomassa não parece ultrapassar um certo limite, mas a produção continua grande, esta massa deve ser deslocada para algum lugar. Três poderiam ser os destinos mais prováveis dela: consumo por animais; acumulação de massa morta sobre o sedimento; e flutuação e deslocamento com o fluxo de água. Em geral, as macrófitas aquáticas não são muito predadas, embora E. densa seja consumida por peixes (Pine \& Anderson, 1991; Crutchfield et al., 1992; Lake et al., 2002; Miyazaki \& Pitelli, 2003). Em todas as coletas do presente estudo, pouquíssimas foram as vezes em que foram encontradas marcas de predação nas plantas. Há alguma acumulação de matéria orgânica no sedimento, porém nunca foi encontrada uma massa grande de pedaços de E. densa mortos e em decomposição. Sabe-se que uma certa quantidade de pedaços de E. densa bóia e atinge as grades das turbinas, mas não se sabe que proporção do estoque de biomassa toma esse destino.

A principal conclusão do trabalho é que a rápida recolonização e o grande crescimento da E. densa tornam muito dificil erradicá-la

Planta Daninha, Viçosa-MG, v. 23, n. 2, p. 363-369, 2005 
dos reservatórios do São Francisco. Mesmo que um esforço gigantesco fosse feito para arrancar as plantas de uma grande área, as plantas que sobrassem levariam a uma recolonização em uma questão de meses ou, no máximo, alguns anos, se a proporção de plantas restantes no reservatório fosse muito pequena. A capacidade de $E$. densa de ocupar completamente um lago, em poucos anos, a partir de uma pequena infestação, já foi comprovada, mesmo em condições de crescimento menos favoráveis que as do São Francisco (Wells \& Clayton, 1991).

Assim, na situação presente, a convivência com a extensa cobertura de E. densa parece inevitável; não resolve o problema da acumulação de partes da planta nas grades de proteção das turbinas, mas pode significar o aproveitamento de um potencial de produção de biomassa em uma região que apresenta grande limitação nesse sentido. $\mathrm{O}$ aproveitamento da produção de $E$. densa tem ainda a vantagem de poder ser feito em qualquer época do ano. Caso a biomassa pudesse ser usada como ração animal, a produção na época seca poderia aliviar a deficiência regional de forragem nesse período. É uma possibilidade que vale a pena ser testada.

A rápida regeneração indica que a eliminação de $E$. densa através da sua retirada dos prados não seria fácil; por outro lado, mostra um grande potencial de produção de biomassa que pode ter uma influência decisiva no ecossistema e que poderia ser explorado comercialmente, caso a planta tivesse algum uso.

\section{AGRADECIMENTOS}

À equipe técnica do Projeto "Estudo do ecossistema dos reservatórios das barragens do sistema hidroelétrico de Paulo Afonso e Itaparica (Terceira Etapa)", na pessoa da Dra. Sônia Maria Barreto Pereira (coordenadora), pela valorosa contribuição logística e financeira. À Companhia Hidroelétrica do São Francisco (CHESF), pelo apoio logístico e acesso aos reservatórios do Sistema Hidroelétrico de Paulo Afonso, BA. Ao Programa de PósGraduação em Botânica da Universidade Federal Rural de Pernambuco, pelo acesso às suas instalações.

Planta Daninha, Viçosa-MG, v. 23, n. 2, p. 363-369, 2005

\section{LITERATURA CITADA}

BARRETO, R. et al. Biological control of neotropical aquatic weeds with fungi. Crop Protec., v. 19, p. 697-703, 2000 .

BINI, L. M. et al. Aquatic macrophyte distribution in relation to water and sediment conditions in the Itaipu reservoir, Brazil. Hydrobiologia, v. 415, p. 147-154, 1999.

BORGES NETO, C. R.; PITELLI, R. A. Adjuvantes e herbicidas e a infectividade de Fusarium graminearum, agente potencial de biocontrole de Egeria densa e Egeria najas. Planta Daninha, v. 22, p. 77-83, 2004.

CAMARGO, A. F. M.; ESTEVES, F. A. Influence of water level variation on biomass and chemical composition of the aquatic macrophyte Eicchornia azurea (Kunth) in an oxbow lake of the Rio Mogi-Guaçu (São Paulo, Brasil). Arch. Hydrobiol., v. 135, p. 423-443, 1996.

COOK, C. D. K.; URMI-KÖNIG, K. A revision of the genus Egeria (Hydrocharitaceae). Aquatic Bot., v. 19, p. 73-96, 1984.

CRUTCHFIELD, J. U. et al. Establishment and impact of redbelly tilapia in a vegetated cooling reservoir. J. Aquatic Plant Manag., v. 30, p. 28-35, 1992.

ESTEVES, F. A. Biomass and analysis of the major inorganic components of floating aquatic macrophytes Eicchornia crassipes (Mart.) Solms in six reservoirs of São Paulo State, Brazil. Ci. Cultura, v. 34, p. 1197-1200, 1982.

FEIJOÓ, C. S. et al. Factors influencing biomass and nutrient content of the submersed macrophyte Egeria densa Planch. in a pampasic stream. Hydrobiologia, v. 341, p. 21-26, 1996.

GANTES, H. P.; CARO, A. S. Environmental heterogeneity and spatial distribution of macrophytes in plain streams. Aquatic Bot., v. 70, p. 225-236, 2001.

GETSINGER, K. D.; DILLON, C. R. Quiescence, growth and senescence of Egeria densa in lake Marion. Aquatic Bot., v. 20, p. 329-338, 1984.

HARAMOTO, T.; IKUSIMA, I. Life cycle of Egeria densa Planchon, an aquatic plant naturalized in Japan. Aquatic Bot., v. 30, p. 389-403, 1988.

HOFSTRA, D. E. et al. Competitive performance of Hydrila verticillata in New Zealand. Aquatic Bot., v. 63, p. 305-324, 1999.

HOFSTRA, D. E.; CLAYTON, J. S. Evaluation of selected herbicides for the control of exotic submerged weeds in New Zealand: I. The use of endothall, triclopyr and dichlobenil. J. Aquatic Plant Manag., v. 39, p. 20-24, 2001. 
HOWARD WILLIANS, C.; SCHWARZ, A. M.; REID, V. Patterns of aquatic weed regrowth following mechanical harvesting in New Zealand hydro-lakes. Hydrobiologia, v. 340, p. 229-234, 1996.

KAHARA, S. N.; VERMAAT, J. E. The effect of alkalinity on photosynthesis - light curves and inorganic carbon extraction capacity of freshwater macrophytes. Aquatic Bot., v. 75, p. 217-227, 2003.

LAKE, M. D. et al. Consumption of submerged aquatic macrophytes by rudd (Scardinius erythrophtalmus L.) in New Zealand. Hydrobiologia, v. 470, p. 13-22, 2002.

LARA, M. V.; CASATI, P.; ANDREO, C. S. $\mathrm{CO}_{2}^{-}$ concentrating mechanisms in Egeria densa, a submersed aquatic plant. Physiol. Plant., v. 115, p. 487-495. 2002.

MABERLY, S. C.; MADSEN, T. V. Freshwater angiosperm carbon concentrating mechanisms: processes and patterns. Funct. Plant Biol., v. 29, p. 393-405, 2002.

MARCONDES, D. A. S. et al. Eficiência de fluridone no controle de plantas aquáticas submersas no reservatório de Jupiá. Planta Daninha, v. 21, p. 69-77, 2003. (Edição especial)

MENDES, D.; PITELLI, R. A.; COELHO, L. Efeito de concentrações de herbicidas sobre aspectos biológicos de Fusarium sp. (isolado FCAV\#940). Planta Daninha, v. 22, p. 85-93, 2004.

MENEZES, C. F. S. Biomassa e produção primária de 3 espécies de macrófitas aquáticas da represa do Lobo (Broa, SP). 1984. 253 f. Dissertação (Mestrado em Ecologia de Recursos Naturais) - Universidade Federal de São Carlos, São Carlos, 1984.

MENEZES, C. F. S.; ESTEVES, F. A.; ANÉSIO, A. M. Influência da variação artificial do nível d'água da represa do Lobo (SP) sobre a biomassa e produtividade de Nymphoides indica (L.) O. Juntze e Pontederia cordata L. Acta Limnol. Bras., v. 6, p. 163-172, 1993.

MIYAZAKI, D. M. Y.; PITELLI, R. A. Estudo do potencial do pacu (Piaractus mesopotamicus) como agente do controle biológico de Egeria densa, Egeria najas e Ceratophyllum demersum. Planta Daninha, v. 21, p. 53-59, 2003. (Edição especial)
NAKANISHI, M.; SARACENI, C.; KURATA, A. Comparison of some limnological variables in the waters between the upper and lower littoral areas within an Egeria stand. Arch. Hydrobiol., v. 116, p. 313-331, 1989.

NASCIMENTO, P. R. F. Produção de biomassa de Egeria densa Planchon, nos reservatórios da Hidroelétrica de Paulo Afonso - Bahia. 2002. 46 f. Dissertação (Mestrado em Botânica) - Universidade Federal Rural de Pernambuco, Recife, 2002.

NEGRISOLI, E. et al. Degradação de diquat em condições de caixa d'água com e sem plantas de egéria. Planta Daninha, v. 21, p. 93-98, 2003. (Edição especial)

PINE, R. T.; ANDERSON, L. W. J. Plant preference of triploid grass carp. J. Aquatic Plant Manag., v. 29, p. 80-82, 1991.

POOVEY, A. G.; GETSINGER, K. D. Impacts of inorganic turbidity on diquat efficacy against Egeria densa.

J. Aquatic Plant Manag., v. 40, p. 6-10, 2002.

TANNER, C. C.; CLAYTON, J. S.; WELLS, R. D. S. Effect of suspended solids on the establishment and growth of Egeria densa. Aquatic Bot., v. 45, p. 299-310, 1993.

TAVECHIO, W. L. G.; THOMAZ, S. M. The effects of light on the growth and photosynthesis of Egeria densa Planchon. Brazil. Arch. Biol. Technol., v. 46, p. 203-209, 2003.

WELLS, R. D. S.; CLAYTON, J. S. Submerged vegetation and spread of Egeria densa Planchon in lake Rotorua, Central North Island, New Zealand. N. Z. J. Marine Freshwater Res., v. 25, p. 63-70, 1991.

WILCOCK, R. J. et al. The influence of aquatic macrophytes on the hydraulic and physico-chemical properties of a New Zealand lowland stream. Hydrobiologia, v. 416, p. 203-214, 1999.

WINTON, M. D.; CLAYTON, J. S. The impact of invasive submerged weed species on seed banks in lake sediments. Aquatic Bot., v. 53, p. 31-45, 1996. 